\title{
Metallography and Microstructures of Metals Used in Medical Devices
}

\author{
G. F. Vander Voort
}

Buehler Ltd, 41 Waukegan Rd, Lake Bluff, Il 60044 USA

Metallic alloys have been used in various forms in medical implants in the human body for many years, although the number of implanted devices has grown markedly over the past twenty years. Early devices employed carbon steels and stainless steels. Carbon steels have not been used in many years and stainless steels have been widely replaced by other alloys, partially due to toxicity problems due to the nickel in these alloys. Low-Ni and Ni-free alloys are currently being developed and introduced. Nitinol, although it contains substantial Ni, is used in certain applications where the shape-memory effect can be utilized and tolerance to $\mathrm{Ni}$ is not a problem. Cobalt-based alloys are being used more frequently due to their high corrosion resistance. Titanium and certain Ti alloys are widely used, as they are inert, and lightweight. There has been an increase in the use of certain refractory metals, $\mathrm{Zr}$, Ta and $\mathrm{W}$, for example, as they have good corrosion properties. Limited use of certain precious metals (aside from dental applications) is also made. This talk discusses specimen preparation and etchants to reveal the microstructure of most of these metals and alloys.

Specimen preparation for studying the microstructure of medical devices, or the raw material used to make these devices, must be carefully performed in order to reveal their structure. While stainless steels are relatively simple to prepare, most of the other metals and alloys are more difficult to prepare perfectly. Nearly all of these metals and alloys require use of specialized reagents to properly reveal the structure. While bright-field illumination is most commonly utilized, other illumination methods are highly useful. For example, commercial purity titanium and zirconium can be examined un-etched using crossed polarized light. Polarized light image quality is dependent upon the elimination of preparation damage and upon the quality of the microscope optics.

Specimen preparation methods for these metals have been developed [1] that yield excellent results using straightforward methods that generally require less than about twenty-five minutes. Automated preparation equipment is recommended as the methods are performed accurately and reproducibly. Manual preparation cannot produce as good edge retention as automated processing and is less reproducible.

Successful preparation requires that sectioning be performed with equipment and consumables that minimize damage. Sectioning is a violent process and it can introduce massive damage. Crystal structure does influence damage depth. Face-centered cubic metals are very ductile as they have twelve excellent slip systems based on three close-packed directions and four close-packed planes. Body-centered cubic metals are less ductile as they have only one close-packed direction and no close-packed planes. Hexagonal close-packed metals deform by mechanical twinning and their ductility is better than bec metals but not as good as fcc metals. Use only abrasive blades designed for metallography and are recommended for the specific metal/alloy in question. A precision saw yields even less damage as the blades are much thinner and the applied loads are much lower.

Mount specimens for best results, especially edge retention. All mounting materials are not equal; each has various benefits and limitations. The least expensive resins are usually not the best 
performing resins, unfortunately. If the device has material diffusion bonded to the surface that is to be against bone, to enhance bone in-growth, it may be advisable to mount the specimen in a cast epoxy resin. But, use a low viscosity resin that can be vacuum impregnated, such as Epo-Heat ${ }^{\mathrm{TM}}$ epoxy. An alternative is to use a hot-compression mounting resin. For such a specimen, add very fine EpoMet ${ }^{\circledR} \mathrm{F}$ epoxy thermosetting resin around the specimen and then put the coarser EpoMet $\mathrm{G}$ resin above that. The fine resin will flow better into the crevices and provide excellent edge retention. If the resin chosen produces shrinkage gaps between specimen and mounting compound, it may be impossible to obtain decent edge retention and bleed out and contamination problems will be encountered.

If the specimen is properly cut with minimal damage, a reasonably flat, smooth surface usually is produced. Commence grinding with the finest possible abrasive that will make all of the specimens in the holder co-planar and remove the sectioning damage in reasonable time. One grinding step is all that is usually needed. Then, follow the recommended preparation methods [1]. These methods utilize flat, woven cloths or pads that minimize relief problems. Charge the cloth with diamond paste of the recommended size and work the paste into the cloth with your fingertip. Add a lubricant that actually provides lubrication, that is, a petroleum-based product. It is imperative that during polishing the cloth surface is covered consistently with the desired abrasive and lubricant. Contra rotation, where the platen and holder rotate in opposite directions, helps keep the cloth surface uniformly covered with abrasive and lubricant if the head speed is $<100 \mathrm{rpm}$. With complementary rotation (platen and holder both rotating counterclockwise), centrifugal force throws the abrasive and lubricant off the cloth and down the drain as fast as it is added, especially when the head speed is $>100 \mathrm{rpm}$. Use the recommended applied loads. Too low a load may introduce relief, especially in final polishing with colloidal silica or alumina slurries. Excessive loads may introduce deformation.

After preparation, the hcp metals can be studied using polarized light without etching. Examine all specimens as polished using bright-field illumination to detect nonmetallic inclusions, nitrides, cracks, surface defects (seams, laps, etc.), and voids. These may be more difficult to observe after etching. Then, select a general-purpose etchant to see what the structure is. If image analysis is desired, it may be necessary to remove this etch (repeat the last preparation step) and etch with a selective reagent [2] to color or darken a specific phase, or everything but the phase. This makes detection simpler.

The use of color etchants should be considered as they have numerous technical advantages over standard black \& white etchants. First, they fully reveal the grain structure. If the grains show a wide range of colors, randomly dispersed across the field, then the grains are randomly oriented. However, if there is a narrow range of colors in the grains, a preferred crystallographic texture is present. Color etchants also reveal segregation and deformation from processing better than standard etchants. A comprehensive review of color metallography is given in [3].

[1] G.F. Vander Voort et al., Buehler's Guide to Materials Preparation, Buehler Ltd, Lake Bluff, IL, 2004.

[2] G. F. Vander Voort, "Selective Etching for Phase Identification," Applied Metallography, Van Nostrand Reinhold, NY, 1986, pp. 1-19.

[3] G. F. Vander Voort, "Color Metallography," ASM Handbook, Vol. 9, Metallography and Microstructures, ASM International, Materials Park, Ohio, 2004, pp. 493-512. 